\title{
SIP Signaling Implementations and Performance Enhancement over MANET: A Survey
}

\author{
Mazin Alshamrani \\ ${ }^{1}$ Studies and Decision Support \\ Center, Department for Planning and \\ Development, Ministry of Haj, Saudi \\ Arabia \\ Godwin Ansa \\ ${ }^{3}$ Department of Computer Science, Akwa Ibom State \\ University, \\ Ikot Akpaden, Mkpat Enin, Akwa Ibom State, Nigeria
}

\author{
Zhili Sun \\ ${ }^{2}$ Institute for Communication \\ Systems (ICS), Faculty of \\ Engineering and Physical Science, \\ University of Surrey, Guildford, \\ GU2 7XH, United Kingdom
}

\author{
Feda Alshahwan \\ ${ }^{4}$ Public Authority for Applied Education and Training, \\ College of Technological Studies, \\ Electronics Engineering Department, Computer Section, \\ Kuwait University, Kuwait
}

\begin{abstract}
The implementation of the Session Initiation Protocol (SIP)-based Voice over Internet Protocol (VoIP) and multimedia over MANET is still a challenging issue. Many routing factors affect the performance of SIP signaling and the voice Quality of Service (QoS). Node mobility in MANET causes dynamic changes to route calculations, topology, hop numbers, and the connectivity status between the correspondent nodes. SIP-based VoIP depends on the caller's registration, call initiation, and call termination processes. Therefore, the SIP signaling performance has an important role for the overall QoS of SIP-based VoIP applications for both IPv4 and IPv6 MANET. Different methods have been proposed to evaluate and benchmark the performance of the SIP signaling system. However, the efficiency of these methods vary and depend on the identified performance metrics and the implementation platforms. This survey examines the implementation of the SIP signaling system for VoIP applications over MANET and highlights the available performance enhancement methods.
\end{abstract}

Keywords-SIP; VoIP; MANET; Peer-to-Peer; Back-to-Back User Agent (B2BUA); IMS

\section{INTRODUCTION}

SIP signaling is widely used to manage and control voice calls over IP-based network systems. The main functions of SIP signaling are: (1) inviting other parties to initiate a call, (2) adding media streams during a call, (3) changing the encoding system during a call, (4) transferring or holding voice calls. The capabilities of the SIP signaling system depends on the implementation systems of the SIP signaling that is used, and the level of support that the network system provides for the application layer services. On the other hand, a Mobile Ad Hoc Network (MANET) is a self-organizing, infrastructure-less, and multi-hop network that consists of unlike groups of nodes with limited capabilities and energy constraints.

The features of MANET include mobility of nodes, variable topology due to the dynamic nature of the network and multipath communication scheme. The communicating nodes in a MANET usually seek the help of other intermediate nodes to establish communication channels. Each node in a MANET works both as a host and a router. Unpredictable connectivity due to the dynamic nature of the network is another challenge faced by MANET. Developing efficient and dynamic routing protocols is a key challenge in MANET.

This review is focused on research in SIP signaling over MANET and the performance enhancement approaches for SIP-based VoIP applications. In this paper, the current stateof-the-art, results, gaps, the merits and demerits of the four types of SIP signaling systems over MANET mentioned here and the performance enhancement methods for SIP signaling over MANET are discussed in detail. Finally, two open issues have been identified and highlighted for future investigations.

\section{A. SIP Signaling System}

SIP is an Internet Engineering Task Force (IETF) standard for signaling protocol released as RFC 3261 [1]. SIP is commonly used for controlling multimedia communication sessions such as voice and video calls over Internet Protocol (IP). SIP is used in initiating, managing, and terminating multimedia sessions such as voice calls over IP based networks. This session can be either a two-way call, which is either unicast or collective multimedia calls, which is multicast. These features have made SIP a better choice for providing VoIP services in the last few years. SIP is an application layer protocol, which serves five main functions for multimedia calls [1]. These functions are: User Location, User Availability, User Capability, Session Setup, and Session Management.

User Location is used to determine the location of the end user, while User Availability examines the willingness of the end user to participate in the call session. User Capability supports the applications compatibility with different communication systems and users to determine the required methods and standards for the requested multimedia 
applications. Session Setup provides the resources to setup and establish the communication. Finally, the Session Management function supports the call management services in different ways such as adding, transferring and modifying the session parameters.

SIP is rather a component which works in a framework with other IETF protocols to build a complete multimedia architecture. The most common protocols which are used in this architecture are: Real-Time Transport Protocol (RTP) for real-time data transportation, Real-Time Streaming Protocol (RTSP) for controlled delivery of streaming media, and Session Description Protocol (SDP) for multimedia session description.

\section{1) SIP Components}

SIP works collectively and in conjunction with different protocols and technologies. SIP consists of two basic components known as User Agents and SIP servers. User Agents are the end points of the call, while SIP servers facilitate the sending of responses back to the requested client. User Agents are self-sufficient in initiating a session with other nodes in the network. Each node consists of two fundamental components known as User Agent Server (UAS) and User Agent Client (UAC). UAC is responsible for initiating a new session, while UAS handles all the connection requests of the clients.

A SIP server is responsible for handling the user name and the IP addresses of the User Agents which connect to it. There are four different SIP servers that are used to handle the calls' interconnection processes to different user agents in the network [2]. These SIP servers are: proxy server, location server, registration server, and redirect server. The proxy server is responsible for forwarding the requests on behalf of user agents. The location server is used to find the information about possible locations for the callee. The location server is most times incorporated within the proxy server features. The address registered to the register server is stored in the location server. The registration server is used for registering a user agent when it is logged into the network.

Hence, registration servers are responsible for registering the location of the user agents. The registration server is used to discover the IP address of the user agents and then map the IP address to the related user name. Finally, the redirect server is responsible for redirecting the clients to the user agents with whom they want to initiate the call session. The redirect server sends back the IP address of the user agent with whom other clients want to communicate. The main difference between the proxy server and the redirect server is that the proxy server forwards on behalf of the $\mathrm{UAC}$, the redirect server on the other hand provides the IP address so that the UAC can contact other UACs directly.

2) SIP Messages

SIP is a text-based protocol similar to the Hyper-Text Transfer Protocol (HTTP), which is used for the forwarding of information between UAC and UAS, by using several requests and responses [3]. The request methods used in SIP are REGISTER, INVITE, OPTIONS, ACK, CANCEL and BYE. The REGISTER request is used for registration when a user agent initially logs on to the network. The INVITE request is used for inviting other UACs to establish communication and then to start a new SIP session between them. The OPTIONS request is used to query the server to find out the capabilities of other User Agents. The ACK request is used to acknowledge a session before exchanging the related messages. The CANCEL request is used to cancel a pending request, while the $\mathrm{BYE}$ request is used in terminating a session. The request methods are replied to with one of the response codes used by SIP.

The request methods used by SIP consist of six classes. The first class of response code belongs to an information or $1 \mathrm{xx}$ which is used to inform that the request is received and processed by having its provisional response, such as 180 ringing. The second class of response code belongs to success or $2 \mathrm{xx}$ which is used for acknowledgment, such as $200 \mathrm{OK}$. The redirection requests or $3 \mathrm{xx}$ is the third class of response code which tells that the request cannot be completed and needs redirection of the user agent, such as 302 moved. The fourth class of request code belongs to client error or $4 \mathrm{xx}$ which signifies that the server cannot process, such as 407. This means that SIP server authentication is required even for the Back-to-Back User Agent (B2BUA) where the SIP server is acting as a UAS. The fifth response class belongs to the server errors or $5 \mathrm{xx}$ which signifies that the server cannot process the request, such as 503 , that means that the service is unavailable. The final class of response code is the server response code, known as the global error or 6xx. This code informs that the server cannot process globally, such as 603, which means decline. When a user agent wants to initiate a session with another user agent, the queries of the client are processed by specific servers.

The proxy-based SIP server on the other hand, relies on the SIP signaling system only for the registration stage of the SIP call processes. This is achieved by maintaining the transaction state of the SIP calls. The IP addresses and locations of the connected clients could be exposed by the callers because the proxy-based SIP server has a low level of security.

Fig. 1 shows the message flow for a simple scenario which depicts the invitation and termination transactions between two users through the B2BUA SIP server. There is a difference between the B2BUA-based SIP server and the proxy-based SIP server with regards to SIP signaling flow. The B2BUA maintains the whole call state and participates in all call requests. It is involved in the call initiation, management, and termination processes. Therefore, the B2BUA system of the SIP server provides a secure, reliable communication system for different User Agents (UAs) where all SIP signaling messages and voice data need to go through the SIP server. 


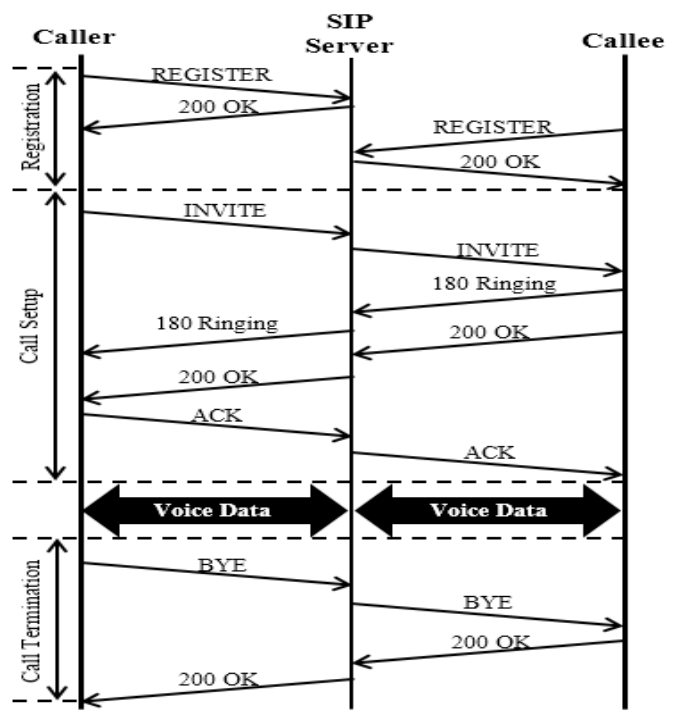

Fig. 1. The signaling flow for a SIP-based VoIP application using B2BUAbased SIP server

The IP addresses, port numbers, and locations of the users are only known to the B2BUA SIP server but hidden from each client thereby providing secure connectivity. The B2BUA SIP signaling system is commonly adopted for privacy approved VoIP implementations, such as military applications and secured call services. The single point of failure problem and congestion overhead are the main disadvantages of a B2BUA-based SIP server. The interactions in Fig. 1 show the use of the SIP methods INVITE, Ringing, and BYE through the SIP Sever. The SIP server depicted here records all the interactions. It is used as the coordinator of the Internet working system between the two ends, with exception of media transmissions. The Media Data mostly depends on the Real-Time Protocol (RTP) and Real-Time Control Protocol (RTCP). The call setup time consumes more time when compared with the termination time. The termination messages could be generated from both ends depending on the type of application and the connection system. In general, the proxy, redirect, register, and location servers are known as the B2BUA SIP Server as represented in Fig. 1. The interactions between the entities of the SIP server are integrated together to provide the SIP services depending on the connectivity methods.

\section{B. SIP Implementations}

Many VoIP phone companies allow clients to use their own SIP devices, as SIP-capable telephone sets, or soft phones. The market for consumer SIP devices continues to expand and there are many devices such as SIP Terminal Adapters, SIP Gateways, etc. The free software community has started to provide more and more of the SIP technology required to build both end points as well as proxy and registration servers. This will lead to a commoditization of the technology and accelerate global adoption. As an example, the open source community at SIP foundry actively develops a variety of SIP stacks, client applications, in addition to entire IP Private Branch Exchange (IP PBX) solutions that compete in the market against mostly proprietary IP PBX implementations from established vendors [4].
SIP-enabled video surveillance cameras can make calls to alert the owner or operator that an event has occurred. For example to notify that motion has been detected out-of-hours in a protected area. Other feasible application examples include video conferencing, streaming multimedia distribution, instant messaging, presence information, file transfer and online games. In general, there are four types of implementations for the SIP signaling system. These implementation types are: Peer-to-Peer SIP system, multiple server based SIP system, single server based SIP system, and IP Multimedia System (IMS) based SIP system.

\section{1) Peer-to-Peer SIP System}

Most of the SIP signaling or traditional SIP signaling is based on Client/Server architecture. In Peer-to-Peer (P2P) architecture, clients have capabilities of both client and server, and are capable of starting a new session with each other and requesting services [1]. Each node is capable of providing services and resources, and in case any node is unable to provide the services then the next node can be contacted. Nodes in a P2P architecture have the features of both UAC and UAS.

Therefore, P2P SIP provides instant messaging or VoIP services with the help of P2P architecture, where session initiation and communication between users is facilitated by the SIP protocol. The Client/Server architecture needs a SIP server for handling requests and responses. However, in the P2P based SIP architecture, there is no need of SIP servers. To-tag, From-tag and Call-ID are collectively used for handling the dialogue between UAC and UAS in P2P-based SIP [5]. Fig. 2 shows message exchange between two devices using P2P SIP.

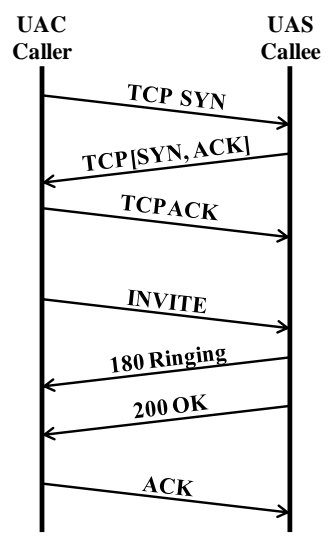

Fig. 2. Signaling flow of messages over Peer-to-Peer SIP

The SIP protocol stack is handled by the various protocols based on the media protocol stack, for example at the transport layer, the TCP/UDP protocol is used. In P2P SIP, two users are involved in the communication process and no SIP server is used as shown in Fig. 2. In this case, users is do not need to register at any SIP server. A TCP SYN packet is sent to open the connection since the TCP protocol is used at the transport layer. SYN consist of an initial sequence number to be used in subsequent communication between the two parties. The callee responds with the SYN message consisting of the initial sequence number and the ACK message, which confirms that 
the callee has received the SYN frame from the UAC. Then the UAC sends the TCP ACK message consisting of the UAC acknowledgement number and completes the 3-way handshake [5].

With the completion of the 3-way handshake, the connection is now open for communication. The UAC caller exchanges the message by sending a SIP INVITE message to the UAS callee. The INVITE message consists of various details, such as session type, which can be either a multimedia or a voice session. There are various other fields in the INVITE message. The first header field in the INVITE message is Via, which is usually a host name and further maps to the IP address using DNS query. In addition, the header field consists of the SIP version, transport layer protocol used, host name and port number. The next header fields are To and From, which dictate the sender and receiver details of the SIP request. Call-ID header field is the next header field which is used to keep track of a particular SIP session [6]. To-tag, From-tag, and Call-ID are known as tags which are collectively used as identifying parameters.

The initial INVITE message consists only of From-tag and the UAC caller generates an INVITE message which consists of both From-tag and Call-ID. In response to the INVITE message, the user agents who respond to this message will generate the To-tag. The SIP parameters From-tag, To-tag, and Call-ID are used to identify an initiated session. Furthermore, the Content-type and Content-length header fields are used to represent the message body as the SDP protocol. The SDP Content-type describes the media information using various SDP fields, such as media format port number, IP address, media transport protocol, media encoding, and sampling rate [6].

After receiving the INVITE message, the UAS callee responds back by sending $1 \mathrm{xx}$ or 180 ringing. The UAS callee creates a 180 ringing message by copying several header fields from the INVITE message [6], such as From, To, and Call-ID. The 180 ringing message consists of a header field known as the CONTACT header field, which specifies an address at which the UAC callee can be contacted. Once the UAC callee is ready to initiate the session, a $200 \mathrm{OK}$ response is sent back to the UAC caller. The $200 \mathrm{OK}$ message consists of the UAS callee SDP message using similar SDP fields. Finally, acknowledgement ACK is sent by the UAC caller to start the media session. Using another protocol for media data transfer, a media session is established between the UAC and UAS. The major advantage of P2P-based SIP is scalability [5]. As in P2P SIP, a user agent need not register with a central server. Instead, the user agent needs to register with an overlay network formed by UAC in the system [5]. Client/Server based SIP needs more maintenance and configuration. On the other hand, P2P-based SIP is more scalable and reliable as there is no single point of failure [7]. In addition, P2P SIP does not need maintenance and configuration including NAT and Firewall. All these benefits come at a cost of increased number of security threats and look-up delays [7]. As in Client/Server based SIP, look-up cost is very low, while in P2P SIP, look-up cost is comparatively very high. Security features such as authentication, and reputation is another major drawback of P2P SIP.

\section{2) Multiple Servers Based SIP System}

The multiple server SIP is based on the client/server architecture in which all the servers, such as proxy server, location server, and registration server, respond to the request sent by the UAC separately. Multiple servers use the Redirect server for initiating a session between a UAC caller and a UAS callee. The Redirect server does not forward the request on behalf of the UAC; it only returns the location shown in Fig. 3. The UAC caller registers itself with the Registration server by sending a REGISTER message. After receiving the REGISTER message from the registration server, it extracts the user name, IP address, and port number then stores them in the location server [6]. A contact header field of the REGISTER message holds information on the lifespan of the registration. Similarly, the UAS callee also registers itself at the registration server. The location details of both the UAC caller and the UAS callee are stored in the location server. An INVITE message is sent by the UAC callee to the redirect server.

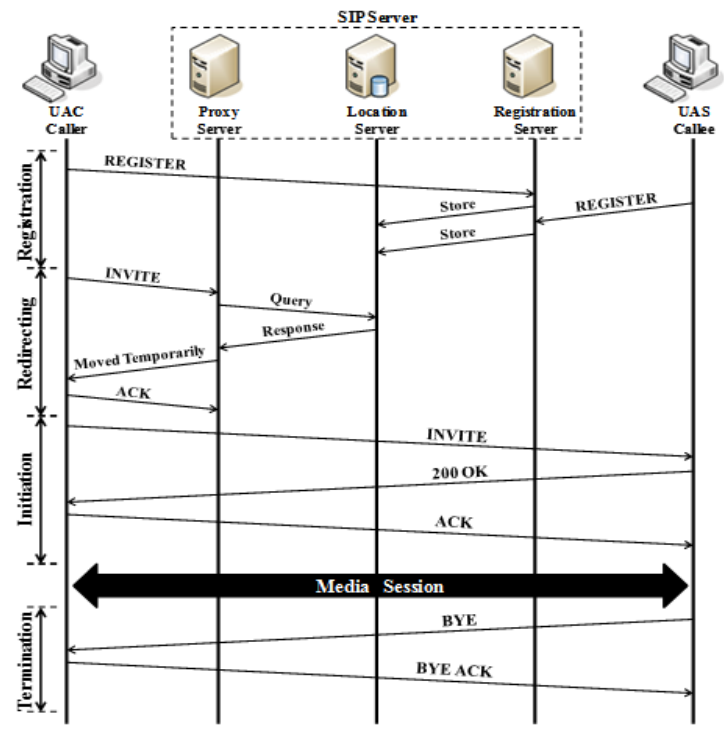

Fig. 3. Signaling flow of SIP messages over multiple SIP servers

The INVITE message consists of the header fields, such as INVITE, Via, Max-Forwards, To, From, Call-ID, CSeq, Subject, Contact-type, and Content-length [2]. The Redirect server performs a look-up within the database of the location server for the intended recipient. Then the location information of the user is sent back to the UAC in a redirection class response. The response Moved Temporarily (302) contains the message format having header fields SIP moved temporarily, Via, To, From, Call-ID, CSeq, Contacttype and Contact-length. After getting the response, the UAC callee acknowledges using an ACK response. At this stage, the redirection process and the exchange process are completed. A new INVITE message is sent directly to the UAS callee as the location is obtained from the control header field of Moved Temporarily in response to the redirect server. The new INVITE message contains a new Call-ID.

In response to the INVITE message, a direct $200 \mathrm{OK}$ response is sent instead of the 180 ringing response. The UAC caller responds to the UAS callee by acknowledging it using 
an ACK response. Thus, a session is initiated between the UAC caller and the UAS callee using a redirect server. After initiating the session, the media session is started between the UAC caller and the UAS callee using the RTP protocol. Once the media session is completed, the session is terminated by sending a BYE request. Once it is acknowledged by the UAC caller, the complete session is terminated. In multiple server based SIP, the redirect server does not forward session initiation requests for the UAC caller as is done by the proxy server. Since the redirect server does not initiate the request, a lower state overhead is needed compared to a proxy server. Multiple server based SIP uses the redirect server which processes very few messages, therefore it has high processing capacity [6].

\section{3) Single Server Based SIP System}

The Single SIP server is based on Client/Server architecture in which the client sends requests to the server, and the server replies to the corresponding request of the client for establishing communication. A UAC requests the services and SIP servers, such as redirect server, or register server respond to those requests. The single server based SIP signaling system is a Back-to-Back User Agent (B2BUS) implementation, as shown in Fig. 1. Initially, the caller sends a REGISTER request to the SIP server. After receiving the REGISTER message, the information in the request message of the caller is updated in the database used by proxies. The REGISTER message sent by a caller consists of the address of the SIP server [6].

The REGISTER request contains To and From header fields. The To header field consists of the User Resource Identifier (URI) to be registered on the server. The next Contact header field containing the SIP URI is stored by the registrar [3]. Then the SIP server acknowledges the caller by sending a $200 \mathrm{OK}$ response message. Similarly, the callee also registers himself on the SIP server. In this case, the SIP server is playing the role of both a registration and location service [6]. After completing the registration process, the caller is not aware of the callee's current location. The caller also needs to check whether the callee is available for the session initiation process or not. Hence, the SIP server is used for inviting the callee, as the SIP server forwards the request on behalf of the user agent. Initially the DNS look-up is performed by the caller SIP URI. It returns the IP address of the SIP server to handle the callee domain. Then the INVITE message is sent to that mapped IP address of the SIP server.

Furthermore, the SIP server looks up in its own database to locate the callee's current location. The process consists of two major steps: the DNS look-up step which is performed by the user agent to find the IP address of the SIP server, then the database look-up which is performed to locate the IP address of the SIP server. An INVITE message is then forwarded by the SIP server to the callee's IP address using a Via header field, having the address of the SIP server [3]. The callee becomes aware that an INVITE message has been routed through the SIP server because the INVITE message consists of two Via header fields. After receiving the INVITE message, the callee sends back a 180 ringing response code to the caller. The 180 response code is created by copying the header fields, such as To, From, Call-ID, and Cseq from the
INVITE request. A response code is sent to the callee through the SIP server. The first Via header field contains the received parameters while the second Via header field contains the IP address in the URI. After receiving the 180 ringing response by the SIP server, the SIP server checks the contents of the first Via header field. Furthermore, when the SIP server finds the first Via header field consists of its own address, it removes the first Via header field and forwards the response to the address within the second Via header field.

Now, the callee is ready to start the session with the caller, it sends back a 200 OK message through the same set of proxies. The SIP server follows a similar process by removing the first Via header field and forwards a $200 \mathrm{OK}$ message back to the caller. The contact header field of the callee in the 200 OK message allows the caller to send an ACK message directly to the callee by bypassing the SIP server. However, it needs to be noticed that the request is sent to the callee's contact URI not in the address of the contact header field. After getting the ACK message from the callee, the session is started between the caller and the callee. At this point, the transmission session is established between the caller and callee using the RTP protocol. In this scenario, the SIP server is used for contacting and locating both end points. The SIP server can drop the path if there is no exchange of media. In the SIP protocol, the path of the signaling message is different from the path of media packets. After the successful transfer of voice data, the connection is terminated using a BYE message. Once the BYE message is received by the callee, it responds by sending back a $200 \mathrm{OK}$ message. On receipt of the $200 \mathrm{OK}$ message, the media session and the transmission process is terminated.

In this case, the SIP signaling is performed using a single SIP server, which forwards the request on behalf of the user agent. A SIP server only forwards the message at the application layer level. It is allowed to modify both request and response, as defined in RFC 3261 [6]. Hence, the SIP server establishes end-to-end communication and preserves end-to-end transparency. As the SIP server can be either a stateful or stateless proxy. All the requests and responses that have been received in the past are tracked by a stateful proxy and can be beneficial for future processing of requests. One such example is the transactional stateful proxy [6]. Reliability is ensured when the TCP protocol is used in a stateful proxy. However, a stateless proxy does not keep track of the request and response messages. A stateless SIP server has higher processing capacity. Major benefits of the SIP server include reliability using replication, flexibility and the use of stateful or stateless proxies. If the number of proxies handling the message exceeds the limits calculated by the Max-forwards header field, then the SIP server discards the messages. If the SIP server is not properly scaled it can have a potential overload

\section{4) IMS-based SIP System}

The IP Multimedia System (IMS) is a concept for providing multimedia services regardless of the media type. The IMS provides a common architectural framework for most media. The IMS consists of multiple SIP proxies known as Call Session Control of Function (CSCF) for supporting multimedia services functionalities. The CSCF with other 
variants, such as P-CSCF (Proxy-CSCF) are used for SIP signaling. The P-CSCF is the first contact point for an IMS terminal and Internet with Gateway GPRS Support Node (GGSN) for resource allocation. The P-CSCF is assigned to an IMS terminal before registration. The I-CSCF (Interrogating$\mathrm{CSCF}$ ) performs similar functions to what the registration server does. The I-CSCF is responsible for routing to the SCSCF. The S-CSCF on the other hand facilitates control and service triggers [8]. The IMS provides more efficient services and provisioning of capabilities than circuit and packet switched networks [14]. When any user initially registers to the IMS, a Subscriber Service Profile (SSP) is downloaded by S-CSCF from a Home Subscriber Server (HSS) [4]. The IMSbased SIP system is shown in Fig. 4.

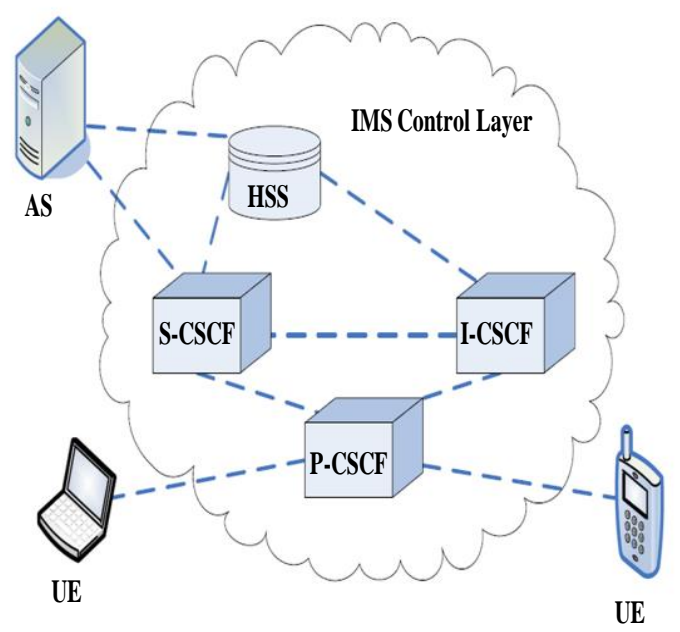

Fig. 4. Signaling flow of SIP messages over IMS-based SIP system

The first step is for the User Equipment (UE) devices to register themselves in the network. Session establishment between UE-1 and UE-2 can be such that either of UE-1 or UE-2 can originate and terminate a session. It is important that a UE has ready resources before sending INVITE and response messages [9]. The SIP-IMS message flow for the initiating session between the two UEs begins from the caller UE-1 to the callee UE-2. Initially UE-1 sends an INVITE message to the P-CSCF. The INVITE message contains various header fields, such as From, To, Call-ID, Cseq, Via, Max-forwards, Route, P-preferred identity, Privacy, Proxyrequire, Security-verify, Contact, Allow, Content-type, and Content-length. After adding itself to record the route header, it forwards an INVITE message to S-CSCF then I-CSCF. The I-CSCF requests the DNS look-up for the location of user UE2 and sends a Location Information Request (LIR) to the HSS. The HSS replies with a Location Information Answer (LIA) by providing the address of the S-CSCF of the terminating subscriber. Then an INVITE message is forwarded to the SCSCF of the terminating visited network. The S-CSCF forwards the INVITE message to UE-2 via the P-CSCF. Then a message, 183 is sent back to UE-1 which indicates the session is in progress. After getting the 183 response code, UE-1 sends a Provisional Acknowledgement (PRACK) to UE2. In responding to the PRACK, a 200 OK message is sent back to UE-1 for Policy Decision Point (PDP) activation, and resource reservation [10].
Next, an UPDATE message from UE-1 to UE-2 and a response code $200 \mathrm{OK}$ is sent back to UE-1 for enabling QoS utilization. Since UE-2 has enough resources readily available, it sends a 180 ringing response to UE-1 via the S-CSCF, I$\mathrm{CSCF}$ and the originating I-CSCF, S-CSCF and P-CSCF. It consists of the header fields, such as From, To, Call-ID, Cseq, Via, Record route, Contact, Privacy, P-Asserted identity, Privacy, and Content-type [4, 10]. UE-1 acknowledges 180 ringing message from UE-2 with a PRACK response. The PRACK consists of header fields, such as From, To, Call-ID, P-Access Network, Cseq, Via, Max-forward, Route, Ack, and Content-length [10]. A 200 OK response is generated and sent back to the UE-1 acknowledging the PRACK request. After acknowledging the PRACK request by an ACK, a session is initiated between UE-1 and UE-2 using the RTP protocol. The IMS SIP has made the provision of services such as multimedia services over IP, VoIP, and IMS possible. It has a very modular design with open interfaces. Hence, it provides flexibility for providing multimedia services over IP networks.

\section{Classification of MANET Routing Protocols}

Routing in MANET is a challenging task as it has a dearth of research efforts. This has led to the development of various routing protocol strategies for MANET. Each new proposed routing algorithm is supposed to be an improved version over some of the previous algorithms, considering the previous literature reviews by the authors. Since each protocol has its pros and cons when comparing it to other protocols, on the basis of certain attributes and different network scenarios. To analyze and compare Mobile ad hoc network protocols therefore, an appropriate categorization method is important. This will be helpful to understanding the nature and distinct properties of available routing protocols.

There are various ways to classify routing protocols in Mobile ad hoc networks. Most of these classifications are done on the basis of certain attributes such as routing strategy and network structure $[11,12]$. Routing strategy is either table driven or source-initiated, so protocols can be categorized as either table-driven protocols or source-initiated protocols. On the structure of the network, protocols are classified as flat routing, hierarchical routing and geographical position as proposed by the authors in [13]. In general, there are three types of routing protocols in MANET [14, 15]:

\section{1) Reactive Routing Protocols}

Reactive routing protocols are on-demand protocols that discover the routes between the source and the destination when needed using the route discovery process. These routes are considered source-initiated. The most widely accepted and used reactive routing protocols are the Dynamic Source Routing (DSR) [16], Ad hoc On-Demand Distance Vector (AODV) [17], Temporally Ordered Routing Algorithm (TORA) [18], and Associativity Based Routing (ABR) [15].

\section{2) Proactive Routing Protocols}

Proactive routing protocols are traditional distributed protocols that use the shortest paths based on periodic updates. Proactive routing protocols are table driven where all possible routes to all destinations are determined at the start. Proactive routing protocols use periodic route updates and have a high routing overhead. The most widely accepted and used 
proactive routing protocols are the Optimized Link State Routing (OLSR) [19], Destination Sequenced Distance Vector (DSDV) [13], Fisheye State Routing (FSR) [20], and Topology Broadcast Reverse Path Forwarding Protocol Fisheye State (TBRPF) [21].

\section{3) Hybrid Routing Protocols}

Hybrid routing protocols have combined functionality from both reactive and proactive routing protocols but possess hybrid routing capabilities. The most widely accepted and used hybrid routing protocol is Zone Routing Protocol (ZRP) [22].

\section{SIP SIGNALING SYSTEM OVER MANET}

An overview of the existing literature on research focusing on SIP signaling performance over MANET and an extensive survey on the related work in this area is presented. This review is mainly focused on research on SIP signaling over MANET and the performance enhancement approaches for SIP-based VoIP applications. Generally, SIP is implemented over MANETs with four different types of SIP signaling systems as represented in Fig. 5.

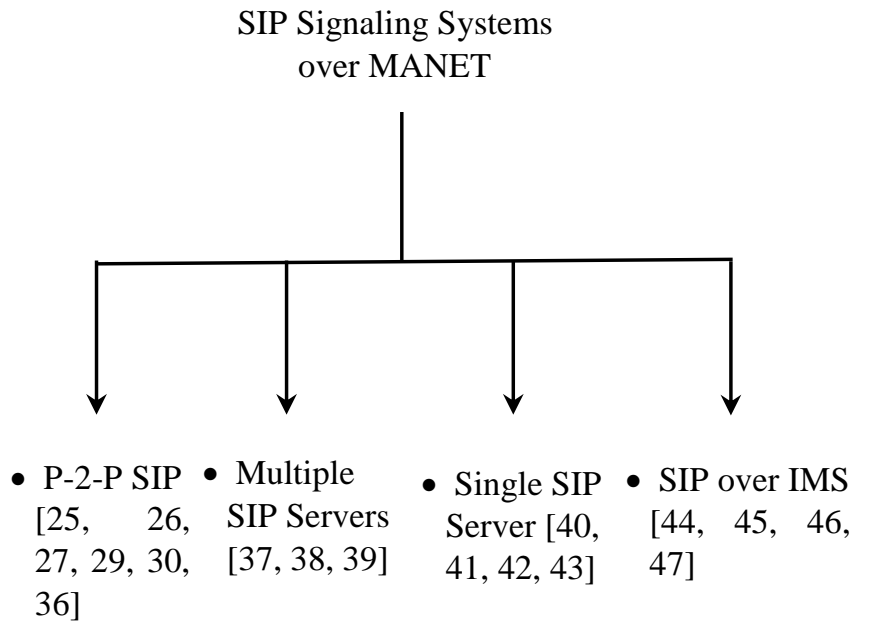

Fig. 5. A survey of types of SIP signaling system implementations over MANET

The first type of SIP signaling system is peer-to-peer SIP over MANET. The main purpose in this case is the elimination of the use of SIP servers. A detailed explanation and review of the existing research of this kind of system is given in section II (A). The second type of SIP signaling system is SIP with multiple servers over MANET. The SIP servers consist of the registration, redirect and proxy servers. The third type of SIP signaling system in the literature is SIP with a single SIP server that acts as a registration, redirect and proxy server over MANET. The fourth type of SIP signaling system over MANET is SIP with an IMS system.

In this section, we will review the current state-of-the-art, results, gaps, advantages and disadvantages with regards to the above-mentioned SIP signaling systems over MANET. Also, the available performance enhancement methods for SIP signaling over MANET will be discussed in section III. Four types for SIP signaling systems and their implementation have been introduced in section I (B). There are a number of research which primarily focuses on adapting SIP to MANETs. Such works can be categorized into two classes. This classification is based on which node(s) act as SIP server(s) in the network.

The first class is characterized with the implementation of the SIP servers in all nodes. Each node can register locally or broadcast location information in the entire network. The second class distinguishes some nodes which act as SIP servers. This survey presents the state-of-the-art in terms of the investigation, evaluation and various service enhancement techniques used in the implementation of SIP signaling system over MANETS. The simulation tools and test-beds for the implementation of SIP signaling systems for MANET will be discussed in this section.

\section{A. Peer-to-Peer SIP Signaling Implementations over MANET}

The authors of [23] propose two solutions for enabling SIP in MANETs: dSIP and sSIP. In dSIP, each node broadcast a REGISTER request to notify all nodes in the network with information about its location. Discovery of members in the network is accomplished by probing the cache locally. To enable Session Initiation Protocol in MANETs, the Service Location Protocol (SLP) [24] is used by sSIP [23]. An SLP request is broadcast from the node that wishes to connect to an ad hoc network in order to ask for bindings of users that are available. Every node that receives an SLP request responds using an SLP reply that includes its binding. As mentioned earlier, using this kind of solution can cause flooding. This can cause problems when used in larger ad hoc networks.

The authors of [25] employ peer-to-peer cover that is structured and related to Chord [26]. In order to map users with the relevant connection information, a Distributed Hash Table (DHT) protocol is used by the nodes. Hence, when some of the nodes connect to the Chord cover, they will be in charge for keeping the information related with the part of the cover that is mapping to its estimated Node-Identification (Node-ID). The maintenance of hash tables contribute to high control overhead. Registration in [27] is achieved by using the multicast mechanism with IPv6. A REGISTER request is multicast by a node to announce its presence to the whole network. The User-List-Cache is updated by each node when REGISTER updates are received. On receipt of the REGISTER updates, each node replies by providing the information to the correspondent using unicast. However, this solution gives poor results and is ineffective for large ad hoc networks, due to the preservation of a User-List-Cache

Research work on the subject of SIP over MANET was initiated in 2003 by the authors in [28]. Their research presents a framework for conference signaling using SIP which allows a MANET user to discover, initiate conferences, and join existing conferences with other users. Another research on SIP over MANET was carried out in 2004 by [29]. In this work, SIP is set up over OLSR using a cross layer, integrated application and routing layer to assist proxy-less and proxy-based systems. A proxy-less system is without a proxy server and a proxy-based SIP MANET contains at least one SIP proxy server. 
Research in the field of proxy-less SIP MANET, i.e. SIP peer-to-peer over MANET without SIP servers is presented in [30]. The authors in this work propose a signaling system that is unique and is used for sessions in $\mathrm{P} 2 \mathrm{P}$ ad hoc networks. Also, the framework proposed in [28] is enhanced in [30] by establishing a hierarchical clustering architecture. This concept is tested through computer emulations on a testbed running on eight computers. The benefit of the proposed system in [30], is that fewer overhead messages are generated when compared to [28].

Most P2P SIP over MANET approaches in the literature use resource discovery mechanisms in order to have the ability to provide SIP user location discovery. Hence, P2P SIP over MANET approaches could be also classified into P2P SIP without overlay network and P2P SIP on an overlay network.

Most approaches on SIP over MANET in the literature employ SIP register and user discovery operations in MANET. These approaches do not deal with the compatibility of their protocols in heterogeneous networks in order to support interoperability between MANET and Internet SIP users. Research solutions for Internet connected MANET environments are presented in [23, 31, 32, and 33]. The proposed solutions rely on a centralized SIP registrar/proxy that can be positioned at the Internet or at the MANET gateway. However, the centralized nature of the registrar/proxy in these solutions creates a traffic bottleneck when SIP requests are sent to the gateway. Another problem is that it creates a single point of failure in the system. With centralized architecture, users SIP binding information are stored by one or a few MANET gateways, called SIP gateways. Another function of a SIP gateway is to forward the received SIP register requests from MANET users to an external SIP registrar on the Internet.

In [27], the authors design and implement the pseudo Session Initiation Protocol (p-SIP) server. The p-SIP server is embedded in each mobile node in order to provide ad-hoc VoIP services. The contribution of this work is two-fold: first, the implemented p-SIP server is compatible with common VoIP user agents. Secondly, it integrates the standard SIP protocol with SIP presence in order to handle SIP signaling and discovery mechanism in the ad-hoc VoIP networks. One advantage is that the implementation of this work is based on real equipment. The implementation of $\mathrm{p}$-SIP is done on IBM ThickPAD x32 laptops, equipped with IEEE 802.11g wireless communication. It uses the Ubuntu Linux 6.10 and Kphone 4.2 as UA which is applied on top of the embedded p-SIP server. With the implementation of the testbed and the performance measurements from the experimental setup, the authors in [27], have shown valuable analysis of the ad-hoc VoIP network.

The results of this work also demonstrate that it is possible to achieve ad-hoc VoIP services using the implemented p-SIP servers. However, the authors did not provide information on different UDP packet sizes, injection rates and contention scenarios. The work however provides information on the influence of TCP/UDP traffic that contend VoIP streams in ad-hoc networks. To improve on the work in [27], further research is needed to analyze the influence of ad hoc node density on performance and the limitation of forwarding hop counts to realize acceptable VoIP QoS in the ad-hoc network.

In [34], the authors suggest a framework for service provisioning in stand-alone MANETs. The contributions of this work provides a new model of business that is harmonized with the features of MANETs. This model allows the invocation and execution of services. It also supports the allocation system of the SIP servlets and overlay networks as a service execution environment. Any user can take part in possessing the required features since the proposed model does not have a central unit and its functionalities. The suggested functional distribution by the authors of this work deals with the number of independent units and the loose coupling.

Also in [34], the authors propose a covering network for execution of stand-alone services in MANETs based on the framework of the SIP servlets. Another contribution of this work is prototypes built to verify ideas for the model of business and the allocated system. This work attempts to prove that the model and the scheme are reasonable with a satisfactory response time. In the results presented, the covering network protocol is formally validated. Though more detailed validation would be needed.

The architecture of a MANET emulator suitable for SIP services is proposed in [35]. The proposed architecture supports real-time audio/video communication, node mobility, and peer-to-peer-type communication. The authors in [35] have developed a SIP_MANET emulator based on the proposed architecture, and it is confirmed that solid communication quality can be maintained with SIP applications. Communication quality evaluation is also conducted to confirm the effectiveness of the simulator. To make achievable usage of the MANET emulator for verifying a SIP application, it is suggested the capabilities to translate the IP address and port numbers be incorporated to give priority to AODV packets, and to process transmission/reception of packets in multiple threads.

When the nodes are stationary, the percentage of successful audio and video communication in a SIP application is approximately $95 \%$. The communication quality in this case is satisfactory. However, when the nodes are mobile, this percentage drops to approximately $77 \%$. It must be noted that multi-path protocols have not been taken into consideration and are not included in the test simulations presented in [35]. Therefore, to enhance the quality of communication when the nodes are mobile, further research on multi-path protocols is needed here.

An innovative Peer-to-Peer (P2P) framework for SIP on MANET is presented in [36]. The focus here is on distributed $\mathrm{P} 2 \mathrm{P}$ resource lookup mechanisms for SIP that tolerate failures resulting from node mobility. The authors of [36] propose a novel P2P lookup architecture based on a Structured Mesh Overlay Network (SMON) that enables P2P applications to perform fast resource lookups in the MANET environment. Their approach extends the traditional SIP user location discovery. It utilizes DHT in SMON in order to distribute SIP object identifiers over SMON. In the simulation conducted, the results show that SIPMON provides the lowest call setup 
delay when compared with the existing broadcast-based approaches. In addition, a new OLSR Overlay Network $(\mathrm{OON})$ is proposed in [36]. The OON is a single overlay network that contains MANET nodes and nodes on the Internet.

The testbed experiment results show that extended SIPMON (SIPMON+) gives better performance in terms of call setup delay and handoff delay when compared with MANET for Network Mobility. Another contribution made in [36] is a proof-of-concept and prototype of P2P multimedia communication based on SIPMON+ for post-disaster recovery missions. This concept is evaluated with experimentation in real disaster situations - Vehicle to Infrastructure scenarios and it is concluded that the proposed prototype outperforms MANEMO-based approaches in terms of packet loss, call setup delay, and deployment time. The proposed framework in [36] can be easily implemented with the day-to-day growth in Internet connectivity. It will be interesting to see more research in this direction to address the issue of how TCPbased applications can be provided on SIPMON+. Session mobility is one issue that need to be investigated and addressed.

\section{B. Implementation of Multiple SIP Servers over MANET}

The authors in [37] propose a distributed protocol called AdSIP that allows SIP implementation in MANETs. This protocol is evaluated on the network simulator ns-2 where comparison is made with the Tightly Coupled Approach (TCA) using metrics such as average session establishment time, failure rate and consumed bandwidth. The evaluation shows that the proposed protocol in [37] has improved performance in terms of adaptability and scalability to node mobility. The proposed solution in [37] chooses a group of nodes that are mobile to act as SIP servers, and they establish a virtual infrastructure as overlay on top of the physical network. A new distributed algorithm is built to construct the topology and to assign dynamically previously explained functionality to a group of nodes in the network. The simulation results obtained using the ns-2 simulator clearly show that the proposed AdSIP protocol is well-adapted to mobile ad hoc network. AdSIP has a lower session establishment time, low control overhead and high service availability.

Apart from the results obtained using the ns-2 simulation tool, this work has not been verified using real results that could be obtained in a real life scenario. Proactive route optimization in SIP mobility is introduced in [38]. The authors' motivation for this work is to achieve latency reduction in session setup. In the proposed Session Initiation Protocol - Proactive Route Optimization (SIP-PRO), the mobility binding information is pre-fetched and used for session establishment during the location registration step. Using the proactive route optimization, reduced latency in session setup is achieved by eliminating the traversal over multiple SIP servers. When a session is initiated, direct establishment of the session with the callee is possible if the caller has valid mobility binding information.

A mobility-aware pre-fetching scheme is developed where only the lower mobility binding information is selected because it is most likely that such information could be used for session establishment. Also in [38], the authors propose a new session setup procedure where mobility information with a sufficient residual time is used. This work lacks extensive simulations using the developed analytical models in order to verify the proposed procedures and optimization level achieved.

\section{Implementation of Single SIP Servers over MANET}

In [39], an intelligent VoIP system with embedded pseudo SIP server in an ad-hoc network is proposed and implemented. The embedded pseudo SIP server presented in this work is compatible with common VoIP user agents using SIP. It acts like middleware between the application and the transport layer. The quality of the VoIP service is evaluated based on the transmission delay for signaling and voice packets. Based on conducted testbed experiments, the results show that an acceptable level of VoIP service quality is achieved. The pseudo SIP server utilizes SIP presence to discover the mobile device and exchange the signaling over an ad-hoc network. This work however lacks some performance metrics such as transmission delay in the experimental results to confirm the quality of the proposed SIP server.

A SIP-based mobile network architecture for Network Mobility (NEMO) in vehicular applications is developed in [40]. The focus of this work is on developing a MANET where the hosts are mobile. Hosts can be either in a vehicle or in a group of vehicles. The MANET is linked to a SIP-based Mobile Network Gateway (SIP-MNG) which connects to the outside. The SIP-MNG is equipped with external wireless interfaces and internal 802.11 interfaces. The SIP-MNG supports call admission control and resource management for the MHs. A boost mechanism with message service that is short has been proposed by the authors. The purpose is to wake up the wireless interfaces in an on-demand manner. The Signaling details of this mechanism is presented in [40]. Additionally, this system is completely well-matched with the SIP standards that are accessible. The prototyping practice and the outcomes of the performance measurements are also presented.

The proposed system saves internet access cost by allowing the sharing of one interface for multiple sessions which is beneficial for both operators and users of public transport. Furthermore, this kind of design supports group mobility where travelers in vehicles could easily access the Internet. A push mechanism which allows SIP-MNG to stay off-line when calling activity is dormant and activates SIPMNG when there is a need is proposed. Maintaining global accessibility of users, the proposed push approach also helps in the reduction of call charges and energy. From the presented experimental results, it is demonstrated that for PHS, WCDMA, and 802.11 networks, it is possible for multiple stations to share one interface. Based on the proposed push mechanism, the call setup time is around 20s. The push server is also designed to select the session temporarily and to use the REFER scheme in order to transmit the session to the client within SIP-MNG. The downside of the proposed mechanism is that the lengthy delay in reconnection time of the wireless interface. Further research is needed in this direction to reduce the reconnection time. 
Converting IP addresses, port number and rewriting SIP messages is required in order to enable a MANET emulator to provide SIP services. However, disruptions may arise between SIP clients, and real-time performance can decline. The authors of [41] propose an architecture for a MANET emulator and local multipath routing appropriate for SIP services. A SIP_MANET emulator is developed and the correct operation of the SIP-VoIP call has been verified. The proposed routing method provides high probability of retaining the required path. The developed system is well described and the evaluation results are presented in detail.

The proposed routing method is compared with AODV and the disjoint multipath routing, using the MANET emulator and the described evaluation model. A measurement of the call holding time is taken. Call holding time is defined as the time from the start of the call to the disconnection of the call is measured. Path retaining probability is also calculated and the effectiveness of the proposed local multipath routing is verified. The proposed routing method uses a spare path when some node in the used path fails. This is the reason why its path retaining probabilities are higher than that of AODV. It would be very useful if the proposed local multipath routing in [41] is compared with AODV on a variety of network models in order to have more detailed results in this domain.

The authors of [42] propose SIPHoc, a middleware infrastructure for session establishment and management in MANETs. SIPHoc is designed to be independent of the underlying network topology, and supports both mobile and static MANETs. Therefore, SIPHoc avoids the problem of having to elect nodes for specialized tasks and replacing them when conditions change. SIPHoc differs from the SIP standard in a fully decentralized implementation which does not require any centralized components, but they both provide the same interfaces.

SIPHoc is message efficient through routing message piggybacking and is independent of the routing protocols. It is also shown that SIPHoc does not impose any topology allowing seamless interaction with the Internet. The architecture, the implementation and performances of SIPHoc are evaluated in [42]. The results show that SIPHoc has a message efficient system and provides a low dial-to-ring delay. In addition, SIPHoc allows the usage of SIP-based applications in MANETs without modification. To support this claim, the authors in [42] show how SIPHoc supports VoIP conversations within MANET, between the end-points and the MANET on the Internet. A VoIP application is used in the evaluation of the performance of SIPHoc to prove that the resulting overhead is near the optimum and comparable with the results of the standard operations on MANETs.

Two approaches enabling SIP-based session setup in ad hoc networks are proposed in [43]. One of them is a loosely coupled method, where endpoint discovery of SIP is decoupled from the procedure of routing. The other approach is the tightly coupled method, which incorporates the endpoint discovery with a cluster supported routing protocol. This protocol is fully distributed and constructs a virtual topology for effective routing. Evaluation through simulation show that the tightly coupled method achieves improved results in terms of latency of the session setup of SIP over static multihop wireless networks compared with the loosely coupled method. On the other hand, results show that the loosely coupled method generally has improved performance in networks that are characterized with random node mobility.

In [43] the authors highlight the problem relating to basic deployment over ad hoc networks and propose solutions for the integration of ad hoc routing protocols with SIP. The use of SIP supported applications for ad hoc networks are not addressed in [43]. However, essential SIP supported session setup for the applications is provided in [43] with no consideration for special applications such as SIP supported conferencing application. Further research is needed to address issues such as load balancing methods and the design and deployment of SIP supported applications.

\section{IMS-based SIP Signaling Implementations over MANET}

IP Multimedia System (IMS) is a developing technology with enormous potential for its usage in MANETs. IMS offers a multimedia Internet experience for different kinds of users using various applications in a mobile environment. The deployment of IMS over MANETs and modern wireless and mobile networks has brought to the fore a plethora of needs and challenges. IMS uses a number of protocols, but its driving force is founded on the SIP. IMS [44] is a 3GPP/3GPP2 standard architecture for the Next Generation Networks (NGN). The goal of this system is to fill the gap that exists between the cellular and the Internet worlds. Hence, IMS offers operators the benefit of the interoperability and quality of telecoms and the modern progress of the Internet [45]. According to the work presented in [46], IMS proposes a SIP servlets-based application server. However, exploiting this technique in MANETs for service provisioning requires a signaling layer. SIP servlets as an option are the best alternative according to the proposed SIP-based architecture for signaling in MANETs in [47].

Three main entities are related to the service provisioning in IMS: HSS, CSCF and the SIP AS. The most important data stored in the HSS are user identities, registration information and security information. The main part represents the user profile. It resolves the services that are offered to each of the users and states the rules for triggering the services. The job of the S-CSCF is to download the user profile or its part from the HSS as soon as the user registers with that S-CSCF for the first time. The S-CSCF also evaluates the initial filter criteria and communicates with the proper application server. Connections between the HSS, the S-CSCF and the AS are achieved with standardized IMS interfaces.

\section{PERFORMANCE ENHANCEMENT APPROACHES FOR SIP- BASED APPLICATIONS OVER MANET}

The current performance enhancement methods for SIPbased applications over MANET vary in terms of system features, requirements, feasibility in implementation, integration with existing systems, and costs. In general, the main performance enhancement methods are related to the dynamic adjustments for SIP timers, dynamic adjustments for the routing protocol parameters, implementations for 
supportive signaling systems, infrastructural based solutions, or service distribution features for the system users.

The dynamic adjustments for SIP timers provide flexible implementation for SIP-based applications over different platforms. This assessment relates to theoretical studies, in reality however, the SIP adjustments need to consider the nature of the network systems that SIP signaling is working on. The wireless and mobility characteristics of MANET affect the SIP signaling performance [40, 46]. Therefore, applying the dynamic adjustments for SIP signaling systems is not a proper solution which can be applied over MANET systems unless the nature of MANET systems had considered this method.

On the other hand, the dynamic adjustments for the parameters of MANET routing protocols have shown an efficient enhancement for different implemented applications. This method depends on accommodating the routing parameters to provide the best level of service for the implemented applications [39]. SIP-based applications using this method show an enhanced level in performance for the SIP signaling and voice data transfer in general [39, 41]. This method is considered as one of the most effective performance enhancement methods. However, no efficient level of implementations has been shown for this method, especially for SIP-based VoIP over MANET for emergency and backup scenarios. The implementation for supportive signaling systems for SIP is considered as one of the effective solutions. Therefore, the SDP signaling system improves the SIP signaling performance over MANET as it supports the management features of the SIP signaling system. However, the lossy nature of MANET is also affects the performance of SDP which increases the performance problems of SIP signaling [41, 48]. Most research studies in the literature implement SIP without SDP.

Synchronization issues between SIP and SDP protocols has been a concern especially the performance of SIP signaling in network systems that are variable in nature or mobility related in their implementation [34, 36, 40, 43]. The infrastructural based solutions use methods to enhance the SIP implementations over MANET. One of the suggested methods implements multiple SIP servers with high performance in order to support larger numbers of MANET nodes [37]. However, this method is difficult to implement for emergency or communication backup scenarios because of the required synchronization functionality between multiple SIP servers for the mobile callers $[39,46]$.

This method could be supported by using the IMS infrastructure since the synchronization functions are secured by its infrastructure. The P2P SIP implementations are considered as the most direct and easiest infrastructural performance enhancement solutions, as described in section I (B.1) [36]. Regardless of the QoS issues, without a central SIP server, it will be difficult to communicate with a large number of MANET-based callers [23]. Other infrastructural methods suggested in the literature include controlling the speed of nodes, limiting the hop numbers, and reducing the background traffic of other simultaneous applications [27, 37, 39].
Other research efforts suggest the use of service distribution features over the system users by scheduling the calls' setup processes. These solutions control the ability of users to initiate voice calls in certain conditions relating to the number of users and amount of bandwidth. The main purpose of these methods is to reduce concurrent calls by applying the time distribution features over the service users to increase the QoS level for the provided services [27, 49]. The merits and demerits of the reviewed performance enhancement methods vary in terms of the enhancement level and implementation requirements. However, both dynamic adjustment for SIP parameters and MANET parameters methods show a good level of performance enhancement. Thus, the most efficient method for enhancing SIP signaling performance over MANET is to qualify the SIP signaling behaviour to conform to the mechanical nature of MANET systems.

Combining both dynamic methods for SIP and MANET has a promising level of performance enhancement with lower costs and simple implementation. However, this enhancement method needs to be based on the evaluation studies for the current state-of-the-art for SIP signaling over different MANET scenarios. In addition, the implementation of these enhancement methods has not been fully investigated over clearly identified mobility models for MANET nodes. The simulation or test-bed tools used do not reflect reliable results that can be considered as reference results for the investigated methods. In addition, none of the proposed solutions in this section have considered any performance metrics for both SIP signaling systems and MANET routing parameters for the SIP-based applications over MANET.

\section{SuPPORTIVE Simulation TOOLS FOR SiP-BASED APPLICATIONS OVER MANET}

The implementation of SIP signaling over MANET protocols as defined in RFC 3261 [50] is available in few number of simulation and test-beds tools. Simulation tools have been used in SIP signaling and MANET research [51]. Although the consistency of the simulation results has been careful analyzed [52]. As a result of this, comparative researches have been published in order to confirm the achieved results [53, 54]. As mentioned in [51], there are a large number discrete-event network simulators that are accessible by the MANET community [55]. From the 80 papers analyzed in [51] and Fig. 1 in [51], [56] we can conclude that the most utilized simulator in MANET research is the Network Simulator-2 (ns-2) with $43.8 \%$ of the analyzed papers. According to this study, there are up to $27.3 \%$ of selfdeveloped simulators

The Global Mobile Simulator (GloMoSim) is used in 10\% of MANET simulations, $6.3 \%$ for QualNet, OPNET® with $6.3 \%$, CSIM simulator with $2.5 \%$ and MATLAB with $3.8 \%$. The OMNeT++ simulator is also used for simulations in MANET. Programs in the simulator are modular in structure. The OMNET++ simulator includes delay as a function of the distance of the nodes. In the ns- 2 simulator, delay is defined as a constant in the configuration file. Because of this, the same kind of parameters will give diverse results although the simulation scenario for MANET could be exactly the same in 
both simulators. MANET can also be simulated with the ns-3 simulator, which is an improved version of the ns- 2 simulator. MANET routing protocols such as Ad-Hoc on Demand Distance Vector (AODV), Destination-Sequenced Distance Vector (DSDV), Dynamic Source Routing (DSR), Optimized Link State Routing (OLSR) can be simulated in ns-3 [57].

The most popular and widely-used network simulators among researchers in MANET and SIP signaling are the ns-2 simulator [58] and OPNET® [59]. There are significant differences at various levels between the two simulators. Consequently, to repeat the results obtained using the ns-2 simulator with the OPNET® simulator, some form of modification is required. Most of the simulation parameters used in ns-2 and OPNET® simulators are the same. However, there are parameters such as the wireless buffer size and the transmission range which are different and influence the simulation results considerably.

For example, if the 802.11 technology with 54 Mbps data rate is used, in ns-2 the default transmission range is 250 meters, while in the OPNET $®$ simulator the default transmission range is 371 meters. Another example worth examining is the buffer size parameter. In the ns-2 simulator, the default buffer size 204,800 bits which is equal to 50 packets (where the size each packet is 512 bytes). In the OPNET® simulator, the default buffer size is 256,000 bits. The differences noticed between these simulators has significant impact on key metrics, like throughput and load.

When these key metrics are processed with the ns-2 simulator, they are computed from the Application level perspective. To be more precise, the presented load is assessed by putting the transmitted data from the application layer on the source node. On the other hand, throughput is calculated by putting up the received data from the application level at a target node. The OPNET ${ }^{\circledR}$ simulator considers metrics such as load and throughput at the MAC level, and that is a reason for two straight outcomes. The first outcome is that overhead is included which is as a result of MAC frame headers, MAC control packets headers, and network protocol headers.

The other consequence is that all the nodes in the network, not just the source and destination nodes are taken into consideration when both statistics are assessed. This means that if any node retransmits packets, the entire load is also increased even when the transmitting node is an intermediate node. In the same vein, when an intermediate node is receiving some packets, the matching cumulative throughput is incremented. Based on these facts, there are differences in the end results. This problem can be solved if a statistic like end-to-end, which is on the Application level similar to the ns2 simulator is assessed.

Another important issue when comparing ns-2 with OPNET $®$ is that error bars are contained in the outcomes from the OPNET® simulator corresponding to the average of $90 \%$ confidence interval. In the ns- 2 simulator, the graphs do not show error bars. The reason is simply because they are not observable, although a confidence interval of $99 \%$ can be reached [53]. This inconsistency is highlighted based on a few constraints that are discovered in the Random Number Generator (RNG) of the OPNET® simulator [60] or weak points of the RNG of the ns-2 simulator [61]. From the above comparison analysis between ns-2 and OPNET®, it can be concluded that the OPNET® simulator performs better and its results more realistic. The reason is that OPNET®, uses approved and supported simulation models and in turn produces more reliable results.

\section{CONCLUSION}

This literature survey has shown that all or some nodes in a MANET have functionalities of SIP, more precisely a proxy and a registrar. User location in SIP could be determined dynamically inside the MANET. With this kind of architecture, SIP implementations over MANET are secured from the single point of failure problem. This is not the case for a centralized SIP architecture. Research in SIP signaling over MANET presented in [23, 28, 29, 31, and 33] lack the inclusion of terminal mobility using SIP. To address this issue, the authors in [36] considered terminal mobility, low call setup delay and fast network operation by proposing the Easy Disaster Communication (EasyDC).

However, another mobility issue, session mobility is not addressed in [36] and should motivate further research. Furthermore, in [23, 28, 29, 31, 32, and 33] every node in a MANET has a role as a SIP register/proxy. Hence, a user of SIP within the MANET can flood the entire network with SIP REGISTER requests in order to register its presence. In contrast to this kind of broadcast-supported SIP register/proxy, is the group-supported SIP register/proxy [30] where registers/proxies take up roles as clusterheads only. These mechanisms utilize flooding SIP requests between the nodes. The result is high network overhead which is a big challenge with the adaptability level of these network systems. In a nutshell, the major problems of SIP signaling over MANET include SIP user lookup time, the mobility assistance of the terminal, and the interoperability between Internet users and SIP over MANET users.

This survey considered possible performance enhancement methods for SIP signaling over MANET. From the literature, it is suggested that enhancing SIP signaling performance is the most efficient method that can be considered when compared to other solutions. In addition, with efficient and simple implementation, combining the dynamic adjustment methods for SIP signaling and MANET routing parameters can improve the performance level.

In this article, we identify the following topics for future investigation to further motivate research interest in SIP signaling over MANET:

1) SIP user lookup time, the mobility assistance of the terminal, and the interoperability between Internet users and SIP over MANET users are some of the challenges SIP signalling over MANET. The use of reliable performance metrics to enhance SIP signalling performance for SIP-based applications over different platforms is still an open research issue which requires further investigation. Performance metrics need to consider the best and worst case scenarios during the dynamic implementation of the SIP signalling system over MANET 
2) Security for SIP-based applications over MANET is another open research issue which needs attention by the research community. There is need to investigate how implementations will cope in the presence of security threats such as Denial of Service (DoS), Man-in-the-Middle and sniffing attacks.

\section{REFERENCES}

[1] J. Rosenberg, et al. "RFC 3261: SIP: Session Initiation Protocol," 2003, URL: http://www.ietf.org/rfc/rfc3261.txt.

[2] H. Sinnreich and A. B. Johnston, "Internet Communications Using SIP: Delivering VoIP and multimedia Services with Session Initiation Protocol," vol. 27. Wiley, 2012.

[3] A. B. Johnston, "SIP: Understanding the Session Initiation Protocol," Third Edition, Artech House Publishers, 2009.

[4] M. Ulvan, A. Ulvan, and R. Bestak, "IMS Signalling in LTE-based Femtocell Network," UBICOMM 2010, The Fourth International Conference on Mobile Ubiquitous Computing, Systems, Services and Technologies. 2010.

[5] G. Camarillo, "Peer-to-Peer (P2P) Architecture: Definition, Taxonomies, Examples, and Applicability," (2009).

[6] A. B. Johnston, "SIP: Understanding the Session Initiation Protocol," Artech House, 2009.

[7] D. Bryan, et al. "Concepts and Terminology for Peer-to-Peer SIP," (2013).

[8] M. Handley, V. Jacobson, and C. Perkins, "SDP: Session Description Protocol," IETF, RFC 4566, Jun 2006, URL: http://tools.ietf.org/html/rfc4566.html.

[9] M. Castro, and A. J. Kassler, "SIP Based Service Provisioning for Hybrid MANETs," Proceedings of International Workshop on Telecommunications, Santa Rita do Sapucaí, Brazil. 2007.

[10] P. Agrawal, et al. "IP multimedia Subsystems in 3GPP and 3GPP2: Overview and Scalability Issues," Communications Magazine, IEEE 46.1 (2008): 138-145.

[11] X. Hong, K. Xu, and M. Gerla, "Scalable Routing Protocols for Mobile Ad Hoc Networks," 2002.

[12] S. Taneja, and A. Kush, "A Survey of routing protocols in mobile ad hoc networks," International Journal of Innovation, Management and Technology 1.3 2010: 2010-0248, 2010.

[13] K. Khan, et al. "An Efficient DSDV Routing Protocol for Wireless Mobile Ad Hoc Networks and its Performance Comparison," Computer Modeling and Simulation, 2008. EMS'08. Second UKSIM European Symposium on. IEEE, 2008.

[14] A. Gupta, H. Sadawarti, and A. Verma, "A Review of Routing Protocols for Mobile Ad Hoc Networks," SEAS Transactions on Communications, 331-340, 2011.

[15] A. Boukerche, et al. "Routing Protocols in Ad Hoc Networks: A survey," Computer Networks 55.13: 3032-3080, 2011.

[16] D. Johnson, Y. Hu, and D. Maltz, "The Dynamic Source Routing Protocol (DSR) for Mobile Ad Hoc Networks for IPv4," Vol. 260. RFC 4728, 2007.

[17] C. Perkins, E. Royer, and S. Das, "Ad hoc On-demand Distance Vector (AODV),” RFC 3561, July 2003.

[18] Z. Zhai, J. Du, and Y. Ren, "The Application and Improvement of Temporally Ordered Routing Algorithm in Swarm Network with Unmanned Aerial Vehicle Nodes," In ICWMC 2013, The Ninth International Conference on Wireless and Mobile Communications, pp. 7-12, 2013

[19] T. Clausen, C. Dearlove, and P. Jacquet, "The Optimized Link State Routing Protocol Version 2," draft-ietf-manet-olsrv2-00, Work in progress, 2006.

[20] N. Sivakumar, and C. Chelliah, "Simulation and Evaluation of the Performance on Probabilistic Broadcasting in FSR (Fisheye State Routing) Routing Protocol Based on random Mobility Model in MANET," Computational Intelligence, Communication Systems and Networks (CICSyN), 2012 Fourth International Conference on. IEEE, 2012.
[21] R. Ogier, F.Templin, and M. Lewis, "Topology Dissemination Based on Reverse-path Forwarding (TBRPF)", IETF RFC 3684, February 2004.

[22] S. Pathak, R. Upadhyay, and U Bhatt, "An Efficient Query Packets Forward Algorithm in ZRP Protocol," Issues and Challenges in Intelligent Computing Techniques (ICICT), 2014 International Conference on. IEEE, 2014.

[23] S. Leggio, and et al. "Session Initiation Protocol Deployment in Ad-Hoc Networks: A Decentralized Approach," 2nd International Workshop on Wireless Ad-hoc Networks (IWWAN). Vol. 5. 2005.

[24] E. Guttman, C. Perkins, J. Veizades, and M. Day, "Service Location Protocol, Version 2," RFC 2165, 1999.

[25] A. O. Driscoll, S. Rea, and D. Pesch, "Hierarchical Clustering as an Approach for Supporting P2P SIP Sessions in Ubiquitous Environments," IFIP/IEEE 9th International Conference on Mobile and Wireless Communications Networks (MWCN), 2007.

[26] I. Stoica, R. Morris, D. Karger, M. F. Kaashoek, and H. Balakrishnan, "Chord: A Scalable Peer-to-Peer Lookup Service for Internet Applications," Proceedings of the ACM SIGCOMM '01 Conference, San Diego, California, August 2001.

[27] L. Chang, C. Sung, S. Chiu, and Y. Lin, "Design and Realization of Ad Hoc VoIP with Embedded p-SIP Server," Journal of Systems and Software, vol. 83, no. 12, pp. 2536-2555, 2010.

[28] H. Khlifi, A. Agarwal, J.-C. Gregoire, "A framework to Use SIP in AdHoc Networks," Canadian conference on electrical and computer engineering, 2003.

[29] L. Li, L. Lamont, "Service Discovery for Support of Real-Time Multimedia SIP Applications over OLSR MANETs," OLSR interop \& workshop, 2004

[30] C. Fu, R. H. Glitho, R. Dssouli, "A Novel Signaling System for Multiparty Sessions In Peer-To-Peer Ad Hoc Networks," IEEE wireless communications and networking conference, 2005.

[31] M. C. Castro, A. J. Kassler, "Optimizing SIP Service Provisioning in Internet Connected MANETs," International conference on software in telecommunications and computer networks, 2006, pp. 86-90.

[32] J. Manner, S. Leggio, K. Raatikainen, “An Internet SIP Gateway for AdHoc Networks," The 3rd annual IEEE communications society on sensor and ad hoc communications and networks, 2006, Vol. 3, pp. 740-745.

[33] P. Stuedi, M. Bihr, A. Remund, G. Alonso, "SIPhoc: Efficient SIP Middleware for Ad Hoc Networks," Proceedings of the ACM/IFIP/USENIX 8th international middleware conference, 2007.

[34] S. Bah, R. Glitho, and R. Dssouli, "A SIP Servlets-based Framework for Service Provisioning in Stand-Alone MANETs," Journal of Network and Computer Applications, 2012.

[35] H. Todoroki, T. Kagoshima, D. Kasamatsu, and K. Takami, "Implementation of a Peer-to-Peer-Type SIP Client Application on a MANET Emulator," in TENCON 2012-2012 IEEE Region 10 Conference, 2012, pp. 1-6.

[36] T. Wongsaardsakul, "P2P SIP Over Mobile Ad Hoc Networks," Institut National des Télécommunications, 2010, PhD Thesis.

[37] S. Yahiaoui, Y. Belhoul, N. Nouali-Taboudjemat, and H. Kheddouci, "AdSIP: Decentralized SIP for Mobile Ad hoc Networks," in Advanced Information Networking and Applications Workshops (WAINA), 2012 26th International Conference on, 2012, pp. 490-495.

[38] S. Pack, P. Jeong, and Y. Kim, "Proactive Route Optimization in SIP Mobility Support Protocol," in Consumer Communications and Networking Conference (CCNC), 2010 7th IEEE, 2010, pp. 1-2.

[39] L. Chang, C. Sung, S. Chiu, and J. Liaw, "Intelligent VoIP System in Ad-Hoc network with Pseudo SIP Server," in Autonomic and Trusted Computing, Springer, 2008, pp. 641-654.

[40] Y. C. Tseng, J. J. Chen, Y. L. Cheng, "Design and Implementation of a SIP-based Mobile and Vehicular Wireless Network with Push Mechanism," IEEE Transactions on Vehicular Technology, vol. 56, No. 6, November, 2007.

[41] T. Kagoshima, D. Kasamatsu, and K. Takami, "Architecture and Emulator in Ad Hoc Network for Providing P2P Type SIP_VoIP Services," TENCON, 2011. 
[42] P. Stuedi, M. Buhr, A. Remund, and G. Alonso, "SIPHoc: Efficient SIP Middleware for Ad Hoc Networks," IFIP International Federation for Information Processing, 2007.

[43] N. Banerjee, A. Acharya, S. K. Das, "Enabling SIP-based Sessions in Ad Hoc Networks," Wireless Networks, Springer, 2007, pp. 461-479.

[44] 3rd Generation Partnership Project, Technical Specifications Group; Services \& Systems Aspects, IP Multimedia Subsystem (IMS), Stage 2, 3GPP TS 23.228 v8.7.0, December 2008.

[45] Ericsson white paper, Introduction to IMS, 284 23-8123 Uen Rrev A., March 2007.

[46] Slimane Bah, "SIP Servlets-Based Service Provisioning in MANETs," A Thesis in the Department of Electrical and Computer Engineering, Montreal, Quebec, Canada, January 2010.

[47] C. Fu, F. Khendek, R. Glitho, "Signaling for Multimedia Conferencing in 4G: the case of integrated 3G/MANETs," IEEE Communications Magazine, August 2006.

[48] S. Massner, C. Richter, and U. Hautzendorfer, "SIP Trunking-General Requirements for Interconnecting Enterprise Networks," Journal of Networks 8, no. 10 (2013): 2195-2212.

[49] M. Voznak, and J. Rozhon, "Methodology for SIP infrastructure performance testing," WSEAS Transactions on Computers 9, no. 9 (2010): 1012-1021.

[50] A. Gupta, H. Sadawarti, and A. Verma, "Review of Various Routing Protocols for MANETs," International Journal of Information and Electronics Engineering 1.3 (2011).99, 2011.

[51] L. Hogie, P. Bouvry, and F. Guinand, "An Overview of MANETs Simulation," Electronical Notes in Theoretical Computer Science, 150, 1, 2006, pp. 81-101.
[52] T. Andel, and A. Yasinsac, "On The Credibility Of MANET Simulations," Computer 39, 7, July 2006, pp. 48-54.

[53] P. P. Garrido, M. P. Malumbres, C. T. Calafate, "ns-2 vs OPNET: A Comparative Study of The IEEE 802.11e Technology on MANET Environments," Simutools '08 Proceedings of the 1st international conference on Simulation tools and techniques for communications, networks and systems \& workshops, 2008.

[54] G. Pandey, S. Kumar, and V. K. Patle, "Comparative Study of Ns2 and OPNET Simulator for AODV and DSR Routing Protocols in MANET," International Journal of Engineering Research and Technology, 2, 7, July 2013.

[55] The SEACORN Project, SEA-CORN Simulation Tools, URL: http://seacorn.ptinovacao.pt/sim_tools.html.

[56] P. Sheeba and C.P. Vandama, "A New SIP-based Application Layer Protocol for VoIP in MANET," International Journal of Engineering Research and Technology, vol. 4, issue 01, pp. 733-737, Jan., 2015.

[57] T. Henderson, ns-3 Tutorial, SIMUTools 2009, http://www.nsnam.org/tutorials.html.

[58] The Network Simulator - ns-2. http://www.isi.edu/nsnam/ns/.

[59] OPNET® Modeler 17.1 Documentation, Oct 2011 Release, OPNET® Technologies. http://www.opnet.com.

[60] M. Becker, T. L. Weerawardane, X. Li, and C. Gorg, "Extending OPNET Modeler with External Pseudo Random Number Generators and Statistical Evaluation By The Limited Relative Error Algorithm," In Proceedings of Recent Advances in Modeling and Simulation Tools for Communication Networks and Services, 2007.

[61] M. Umlauft, and P. Reichl, "Experiences with the ns-2 Network Simulator - Explicitly Setting Seeds Considered Harmful," Wireless Telecommunications Symposium, 2007. 